\title{
Opening Pandora's Box - Presumed ST Segment Elevation Myocardial Infarction with Symptomatic Ventricular Tachycardia is Cured by Resection of a Huge Left Ventricular Aneurysm
}

\author{
Shaojie Chen, MD, PhD, ${ }^{1}$ K.R. Julian Chun, $M D,{ }^{1}$ Thomas Walther, $M D,{ }^{2}$ Tomas Holubec, MD, ${ }^{2}$ \\ Boris Schmidt, $\mathrm{MD}^{1}$ \\ ${ }^{1}$ Cardioangiologisches Centrum Bethanien (CCB), Frankfurt am Main, Medizinische Klinik III, Agaplesion Markus Krankenhaus, \\ Frankfurt am Main, Germany; ${ }^{2}$ Herz-, Thorax- und Thorakale Gefäßchirurgie am Universitätsklinikum Frankfurt, Frankfurt am \\ Main, Germany
}

\section{INTRODUCTION}

Monomorphic ventricular tachycardia is a rare presentation of acute myocardial infarction. Urgent coronary angiography and revascularisation should be part of the management of patients with ventricular arrhythmias [Neumann 2018].

Case report: A 76-year-old male patient with history of hypertension, type 2 diabetes, and smoking was admitted to the emergency department with chest pain. The patient reported severe and sudden chest pain accompanied by cold sweating, dizziness, and palpitations.

As presented in Figure 1, 12 lead-ECG on admission showed monomorphic wide QRS tachycardia with a heart rate of $181 \mathrm{~min}-1$. Due to symptomatic hypotension (68/38 $\mathrm{mmHg}$ ) urgent electrical cardioversion was performed. In normal sinus rhythm, the ECG showed significant ST elevation in the inferior leads. The troponin level was $303 \mathrm{pg} / \mathrm{ml}$ (reference $<14 \mathrm{pg} / \mathrm{ml}$ ).

Due to presumed ST elevation myocardial infarction, immediate coronary angiography was performed. Angiography showed mild to moderate atherosclerosis of the left coronary artery and a proximal occlusion of the right coronary artery (Figure 2).

Left ventricular angiography revealed a huge inferior basal aneurysm $(45 \times 45 \times 35 \mathrm{~mm}$; Figure 3$)$ and a moderately depressed $\mathrm{LV}$ function. After determining the exact diameter of the LV aneurysm and confirming vitality of the apical inferior wall by magnetic resonance imaging, the patient urgently was referred to the heart surgery department.

Surgery included aneurysmectomy using a pericardial patch $(65 \times 45 \mathrm{~mm})$ and a Prolene 3.0 suture (Figure 4). Concomitant mitral valve annuloplasty was performed by implanting a $30 \mathrm{~mm}$ Carpentier Edwards ring. In addition, bypass surgery using a single vein graft to the posterior

Received November 7, 2018; received in revised form February 2, 2019; accepted February 6, 2019.

Correspondence: Boris Schmidt, MD, Cardioangiologisches Centrum Bethanien, Frankfurt am Main, Medizinische Klinik III, Agaplesion Markus Krankenhaus, Wilhelm-Epstein Straße 4, 60431 Frankfurt am Main, Germany; +49-069945028-0 (e-mail:b.scbmidt@ccb.de). interventricular artery was performed. The patient recovered well and presented for follow-up after 90 days with a completely recovered LV function.

\section{DISCUSSION}

Acute myocardial ischemia may trigger ventricular arrhythmias, in particular ventricular fibrillation. The pathophysiology of monomorphic ventricular tachycardia in the setting of ischemic heart disease is usually scar-related reentry rather than a sign of on-going ischemia. In our case, cardiologists were intrigued by the resting ECG pattern compatible with ST segment elevation myocardial infarction. Luckily,
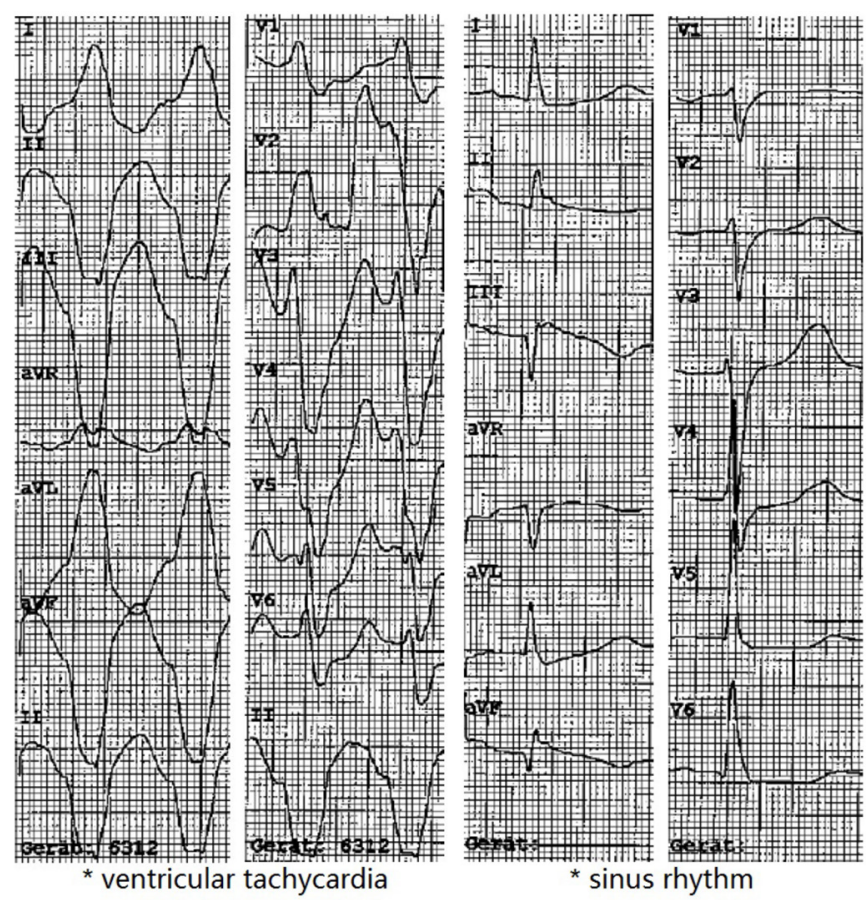

Figure 1. ECGs at admission, ventricular tachycardia, and sinus rhythm after cardioversion 

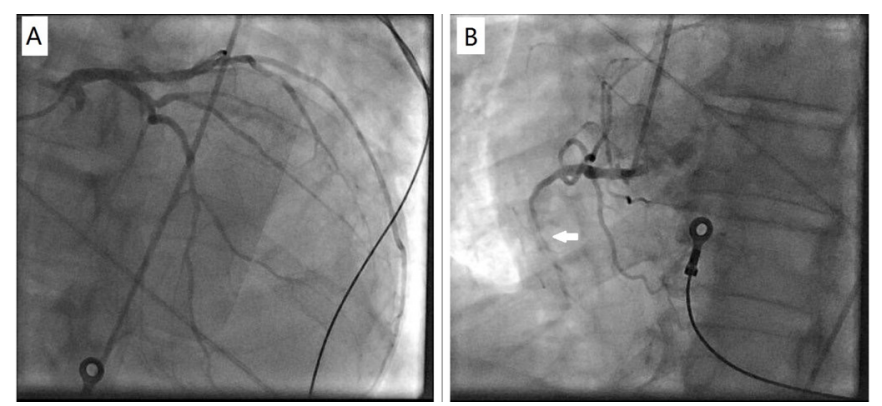

Figure 2. Coronary angiography of the left $(A)$ and right coronary artery (B).

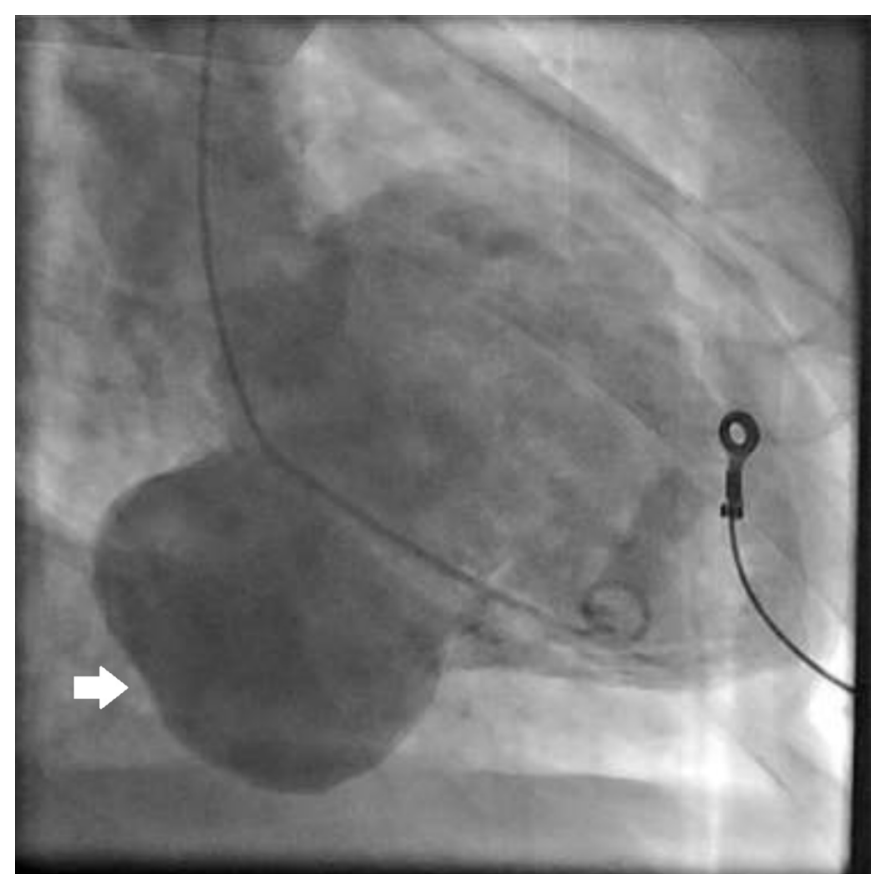

Figure 3. Ventricular angiography shows a tremendous aneurysm.

LV imaging led to the correct diagnosis and paved the way for timely best medical care. Today, large posterobasal aneurysms usually are resected and a patch repair is performed (Dor procedure). In this particular case, annuloplasty was added to achieve optimal stability of the mitral apparatus resulting in
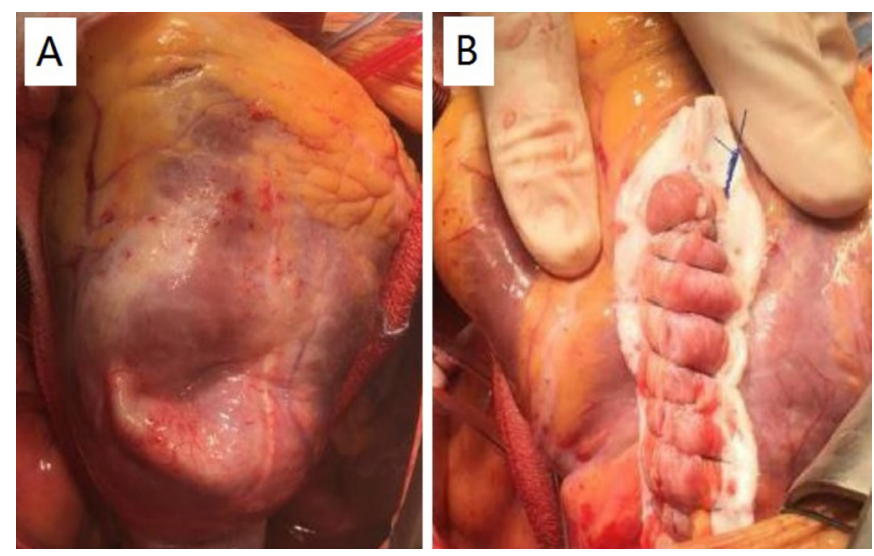

Figure 4. Cardiac surgery shows the ventricular aneurysm and aneurysmectomy.

positive remodeling. The long-term outcome after posterobasal aneurysmectomy is favorable, reporting 10-year survival rates of 53 percent [Toker 2013]. Surgery achieved a significant improvement of left ventricular function and eliminated the arrhythmogenic substrate. Therefore, a mutual decision was taken not to implant an ICD. One might have considered a programmed ventricular stimulation to assess the individual risk for sudden cardiac death.

Moreover, it is debatable whether bypass grafting of the chronically occluded RCA was necessary, but due to the collateral flow to the peripheral RCA and proven vitality in the MRI scan, the operator aimed at the most optimal perfusion.

In conclusion, the present case illustrates the pitfalls of emergency cardiovascular medicine that were subsequently managed in a multidisciplinary approach. Collaborating interventional cardiologists and cardiac surgeons successfully closed Pandora's box.

\section{REFERENCES}

Neumann F-J, Sousa-Uva M, Ahlsson A, et al. 2018. 2018 ESC/EACTS Guidelines on myocardial revascularization. Eur Heart J 1-96.

Toker ME, Onk OA, Alsalehi S, et al. 2013. Posterobasal left ventricular aneurysms: surgical treatment and long-term outcomes. Texas Hear Inst J 40:424-7. 